\title{
Commentary
}

\section{Complex case: A biofeedback intervention to control impulsiveness in a severely personality disordered forensic patient by Rick Howard, Klaus Schellhorn and John Lumsden}

MICHAEL I. CASHER, Department of Psychiatry, University of Michigan Medical School, Michigan, USA

With this case report of successful biofeedback treatment for a severely disordered and treatmentrefractory forensic patient, the authors contribute to arguments for consideration of the inclusion of biofeedback for a group of extremely challenging patients. The term 'complex patient' barely captures the patient $\mathrm{MN}$, whose psychopathology cuts across diagnostic borders and includes a mixed personality disorder with borderline, antisocial and histrionic features, paedophilia, attentional difficulties that may meet criteria for Attention Deficit Disorder/ Attention Deficit Hyperactivity Disorder, borderline intellectual functioning and an unspecified mood disorder. Prior to his transfer from a prison to a forensic hospital, the unremitting severity of his sexual deviance led to placement on a testosteronelowering agent, and his level of mood instability resulted in treatment with carbamazepine.

Strengths of this case report include the extensive initial psychometric and psychological evaluation of the patient and the documentation of significant post-treatment improvement in biobehavioral (Flanker test) and electrophysiological (Go/No Go contingent negative variation) measures, as well as patient self-report of decreased impulsivity and improvement in attention/cognition at the study endpoint and at 3 months of follow-up.

This paper is a welcome addition to the literature on therapeutic interventions in severely personality disordered patients from several perspectives. Much of this patient's history and behavioural presentation are characteristics of antisocial personality disorder (ASPD) and accompanying paedophilia, areas of psychopathology much neglected in psychiatric literature, with diagnoses for which therapeutic pessimism bordering on nihilism has led in the US to relegation of these patients to the criminal justice system. Patients with ASPD have significant deficits in mentalization, attachment and impulse control (Leichsenring et al., 2003), ego weaknesses that make them poor candidates for many psychotherapies and that render these patients destructive to a hospital-based therapeutic milieu. Indeed, MN had shown poor motivation and compliance with routine psychotherapies in the past. An advantage for forensic-type patients of neurofeedback treatments is that they work at basic neurophysiologic levels and focus on brain functioning rather than psychopathology; consequently these procedures largely bypass the need 
for the classic psychotherapeutic alliance. Biofeedback's mechanism of action may actually be altering more basic endophenotypic substrates of impulsivity, represented for instance by electrocortical measurements such as the contingent negative variation. The clinical usefulness of this narrowly defined focus gains support in the finding of lowered electrodermal responsiveness in borderline personality disorder and ASPD, a trait which has been postulated to lead to risk-taking and impulsive behaviours (Herpetz et al., 2001). Furthermore, for confined or incarcerated patients, biofeedback treatments would be less dependent on the therapeutic milieu than traditional psychotherapies.

The possibility that biofeedback could be ameliorative for some of the most problematic manifestations of severely personality disordered patients is an exciting avenue for further exploration. However, because of the naturalistic nature of this study and the presence of several psychiatric co-morbidities in $\mathrm{MN}$, caution is advised in extending the findings from this case study to personality disorders in general. For example, the presence of attentional defects in this forensic patient complicates the understanding of the mechanism of MN's improvement with biofeedback. The evaluation of this patient might have been strengthened by more definitive clinical and neuropsychological evaluation for ADHD/ ADD, as it could be argued that his favourable response to biofeedback represented a positive response in ADHD symptoms. The evidence base for efficacy of biofeedback therapies for ADHD/ ADD is more robust than that for personality disorders (Lubar, 1991; van Outsem, 2011). Rossiter and La Vaque (1995) found significant reductions in behavioural and cognitive symptoms in a group of patients with DSM-defined ADHD treated with 20 sessions of biofeedback over a 4-7-week period. Degree of improvement with biofeedback compared favourably with that seen with the study subjects treated only with psychostimulants. A comprehensive review of neurofeedback therapies in ADHD showed positive outcomes in approximately 80\% of patients (van Outsem, 2011). In his review of biofeedback for forensic patients, van Outsam places biofeedback's evidence for treatment efficacy in ADHD in the highest group (category $\mathrm{A}$ ), along with pervasive developmental disorder/autism and substance abuse disorders, whereas biofeedback for personality disorders does not even warrant inclusion in the lowest evidence group (category $\mathrm{C}$ ). And although van Outsam offers-as pointed out by Howard et al. in the complex case report-that biofeedback could conceivably play a role in the treatment of personality disorders, he prefaces this speculation with the observation that '...there is insufficient evidence that neurofeedback could reduce aggressive or anti-social behaviour in patients suffering from personality disorders.'(van Outsem, 2011)

In summary, by using the case of $\mathrm{MN}$, the authors have stimulated interest in utilizingbiofeedback for a challenging group of patients who are often treated unsuccessfully with medications and/or traditional psychotherapies. The case of $\mathrm{MN}$ illustrates the complexity of these patients with the frequent presence of multiple comorbities. The field of forensic psychiatry could benefit greatly from further study of the use of biofeedback as an addition to its therapeutic armamentarium. Further avenue for clinical research in forensic patients might involve a focus on whether the biobehavioral and neurophysiologic changes translate into improvements in clinical outcome (e.g. length of stay and recidivism).

\section{References}

Herpertz S.C., Werth U., Lukas G., Qunaibi M., Schuerkens A., Kunert H.J., et al. (2001). Emotion in criminal offenders with psychopathy and borderline personality disorder. Archives of General Psychiatry 58(8), 737.

Leichsenring F., Kunst H., Hoyer J. (2003). Borderline personality organization in violent offenders: correlations of identity diffusion and primitive defense mechanisms with antisocial features, neuroticism, and interpersonal problems. Bulletin of the Menninger Clinic 67(4), 314-27

Lubar J.F. (1991). Discourse on the development of EEG diagnostics and biofeedback for attention-deficit/ hyperactivity disorders. Applied Psychophysiology and Biofeedback 16(3), 201-25.

Rossiter D.T.R., La Vaque T.J. (1995). A comparison of EEG biofeedback and psychostimulants in treating 
attention deficit/hyperactivity disorders. Journal of Neurotherapy 1(1), 48-59

van Outsem R. (2011). The applicability of neurofeedback in forensic psychotherapy: a literature review. Journal of Forensic Psychiatry EF Psychology 22(2), 223-42.
Address correspondence to: Michael I. Casher, MD, UH9C 91501500 E. Medical Center Drive, Ann Arbor, Michigan 48109 USA. Email: mcasher@ med.umich.edu 